\title{
Problemas en la red de distribución eléctrica en el distrito de Changuinola, provincia de Bocas del Toro
}

\section{Problems in the electrical distribution network in the district of Changuinola, province of Bocas del Toro}

\author{
Diocelina Guerra ${ }^{l}$, Joustin Saldaña ${ }^{l}$, José Riveral, Joannys González ${ }^{l}$, Vanessa Valdés ${ }^{2 *}$ \\ ${ }^{1}$ Licenciatura en Sistemas Eléctricos y Automatización-Centro Regional de Bocas del Toro- Universidad Tecnológica de Panamá. \\ ${ }^{2}$ Docente Universidad Tecnológica de Panamá, Centro Regional de Bocas del Toro.
}

\begin{abstract}
Resumen La provincia de Bocas del Toro cuenta con dos hidroeléctricas que generan energía eléctrica para toda la población panameña. Sin embargo, en esta provincia se presentan constantes apagones, por lo cual, se ha desarrollado esta investigación con el objetivo de identificar los problemas en la red de distribución eléctrica en el distrito de Changuinola, provincia de Bocas del Toro. Este estudio tiene un enfoque cuantitativo, porque utiliza la recolección de datos con base en la medición numérica y el análisis estadístico. El diseño de la investigación es no experimental, de tipo prospectivo y transversal. Se aplicó un cuestionario de cinco preguntas a una muestra de 380 usuarios, con un nivel de confianza de $95 \%$ y un margen de error de $5 \%$. Los datos fueron analizados obteniendo los siguientes resultados: el $42 \%$ de los usuarios de energía eléctrica afirmaron que sufren de apagones eléctricos cinco veces por mes, y que debido a los apagones las pérdidas materiales andan alrededor de $26 \%$. El $26 \%$ de los usuarios indican que los cables son antiguos, pudiendo ser la causa de los apagones eléctricos. El 26\% de los usuarios piensan que los apagones son causados por eventos naturales, como la caída de árboles, fuertes vientos y lluvias, pero son datos que no pueden asegurar.
\end{abstract}

Palabras clave Distribución eléctrica, voltaje, fluido eléctrico.

\begin{abstract}
The province of Bocas del Toro has two hydroelectric plants that generate electricity for the entire Panamanian population. However, in this province there are constant blackouts, so this research has been developed with the objective of identifying the problems in the electrical distribution network in the district of Changuinola, province of Bocas del Toro. This study has a quantitative approach, because it uses data collection based on numerical measurement and statistical analysis. The research design is non-experimental, prospective and transversal. A questionnaire of five questions was applied to a sample of 380 users, with a confidence level of $95 \%$ and a margin of error of 5\%. The data was analyzed obtaining the following results: $42 \%$ of the users of electric power affirmed that they suffer from electric blackouts five times per month, and that due to the blackouts the material losses are around $26 \%$. 26\% of users indicate that the cables are old and may be the cause of the electrical blackouts. $26 \%$ of users think that the blackouts are caused by natural events, such as falling trees, strong winds and rains, but they are data that cannot ensure.
\end{abstract}

Keywords Electrical distribution, voltage, electric fluid.

* Corresponding author: vanessavvs@gmail.com

\section{Introducción}

La República de Panamá, así como casi la totalidad de los países latinoamericanos, han pasado en el siglo XX, por una evaluación de la administración y estructura del servicio público de electricidad, desde la formación de pequeñas empresas privadas aisladas, seguido por la nacionalización y estatización con la empresa Instituto de Recursos Hidráulicos y Electrificación (IRHE) [1].

En la mayoría de los países, el suministro eléctrico comercial se abastece a través de redes nacionales, que interconectan numerosas estaciones generadoras a las cargas. En Perú [2], se está discutiendo y desarrollando la definición de mecanismos reguladores y condiciones técnico-económicas de conexión de generación distribuida, para lograr que los usuarios finales con bajo consumo de energía tengan menos incentivos para convertirse en prosumidores, debido a la existencia de una tasa subvencionada.

Según [3], en los sistemas de distribución interesan la confiabilidad de operación, para mejorar el servicio a los clientes y evitar multas, y la confiabilidad de planificación, para diseñar redes eléctricas con poca probabilidad de falla 
La República de Panamá que cada día avanza y se vuelve más tecnológico; se ha vuelto más dependiente de la disponibilidad continua de suministro eléctrico [4]. En Panamá el suministro eléctrico comercial y residencial se abastece de una red nacional de distribución centralizada dirigida por el Centro Nacional de Despacho, el cual, es una dependencia de la Empresa de Transmisión Eléctrica S.A.(ETESA). Esta se encarga de coordinar las operaciones y las transacciones que se dan entre los participantes del mercado mayorista de electricidad.

La red de distribución debe abastecer las necesidades básicas nacionales de iluminación, calefacción, refrigeración, aire acondicionado, transporte y residenciales, así como el abastecimiento crítico a comunidades gubernamentales, industriales, financieras, comerciales, médicas y de comunicaciones. El suministro eléctrico comercial literalmente le permite al mundo moderno funcionar a paso acelerado. La sofisticada tecnología ha penetrado profundamente en los hogares e instituciones gubernamentales y no gubernamentales, y la llegada del comercio eléctrico está cambiando continuamente la forma en la que se interactúa con el resto del mundo.

Los sistemas de distribución constituyen una parte fundamental en la prestación y en el aseguramiento de la calidad del servicio eléctrico. Considerando los cuatro (4) procesos fundamentales como la generación, transmisión, distribución y comercialización, los sistemas de distribución son los que contabilizan la mayor cantidad de activos instalados siendo estos la longitud de línea, número de estructuras de soporte, elementos de maniobra y transformadores, entre otros [5].

El distrito de Changuinola se encuentra ubicado a $332.9 \mathrm{~km}$ lineales y más de $600 \mathrm{~km}$ de carretera a partir del kilómetro cero, de la Ciudad de Panamá (Changuinola Coordenadas GPS: 9.43,-82.52 - Ciudad de Panamá Coordenadas GPS: 8.9936,79.51973) [6], en donde se ubica el Centro Nacional de Despacho (CND). En el CND comienza el proceso de distribución de energía eléctrica a todas las partes del país. Con el avance de las tecnologías, la mayoría de los equipos eléctricos y electrónicos de uso doméstico e industrial son más vulnerables a variaciones de los parámetros de alimentación. Por ende, la calidad de la energía y la continuidad en el servicio de suministro, deben regirse bajo principios de confiabilidad.

En el distrito de Changuinola se sufre a diario de interrupciones y fallas en las líneas de distribución y trasmisión de energía eléctrica, siendo estas causantes de pérdidas económicas y sociales asociadas a los factores característicos de la energía eléctrica.

El gobernador de la provincia de Bocas del Toro, Ubaldo Vallejos [7], asegura que el problema del suministro de energía es delicado, sin embargo, en conversación con el gerente general de la OER, se concluyó que de los fondos del gobierno central se asignarían 300 mil dólares para la compra de equipos como transformadores, cables y herramientas para que los técnicos puedan realizar sus labores de manera segura [8].

A partir de la investigación desarrollada se busca identificar la problemática causante de los fallos en la discontinuidad en el servicio eléctrico en Changuinola, por lo cual, surge la siguiente interrogante, ¿Cuál es la problemática en la red de distribución eléctrica en el distrito de Changuinola?, considerando así, el siguiente objetivo: Identificar las posibles causas de las fallas e interrupciones en la red de distribución eléctrica en el distrito de Changuinola.

\section{Materiales y Métodos}

\subsection{Tipo de estudio}

Este estudio tiene un enfoque cuantitativo, porque utiliza la recolección de datos para probar hipótesis con base en la medición numérica y el análisis estadístico, con el fin de establecer pautas de comportamiento y probar teorías [9]. El enfoque cuantitativo es secuencial y probatorio. Cada etapa precede a la siguiente y no se puede "brincar" o eludir pasos.

El diseño de investigación es no experimental, porque se realiza sin manipular deliberadamente variables, es sistemática y empírica en donde las variables no se manipulan. Las inferencias sobre las relaciones entre variables se realizan sin intervención o influencia directa, y dichas relaciones se observan tal como se han dado en su contexto natural [9].

Según [9] el tipo de estudio es prospectivo, ya que, está dirigido a responder las causas de los eventos y fenómenos físicos o sociales. Se enfoca en explicar por qué ocurre un fenómeno y en qué condiciones se manifiesta, o por qué se relacionan dos o más variables, y en este caso, las constantes interrupciones y fallas en la red de distribución de energía eléctrica en Changuinola. También es transversal, puesto que, se recolectan datos en un solo momento, en un tiempo único [9]. Su propósito es describir variables y analizar su incidencia e interrelación en un momento dado; es como "tomar una fotografía" de algo que sucede.

\section{2 Área de Estudio}

Esta investigación se desarrolló en el distrito de Changuinola, Provincia de Bocas del toro, República de Panamá, ver figura 1.

Bocas del Toro es una provincia de Panamá y su capital es la ciudad homónima de Bocas del Toro. Tiene una extensión de $45843,9 \mathrm{~km}^{2}$, una población de 125,461 habitantes (2010) y sus límites: al norte con el Mar Caribe, al sur con la provincia de Chiriquí, al este y sureste con la Comarca Ngäbe-Buglé, al oeste y noroeste con la provincia de Limón de Costa Rica; y al suroeste con la provincia de Puntarenas de Costa Rica. La provincia incluye la isla Escudo de Veraguas que se encuentra 
en el Golfo de los Mosquitos y separada del resto por la Península Valiente [10].

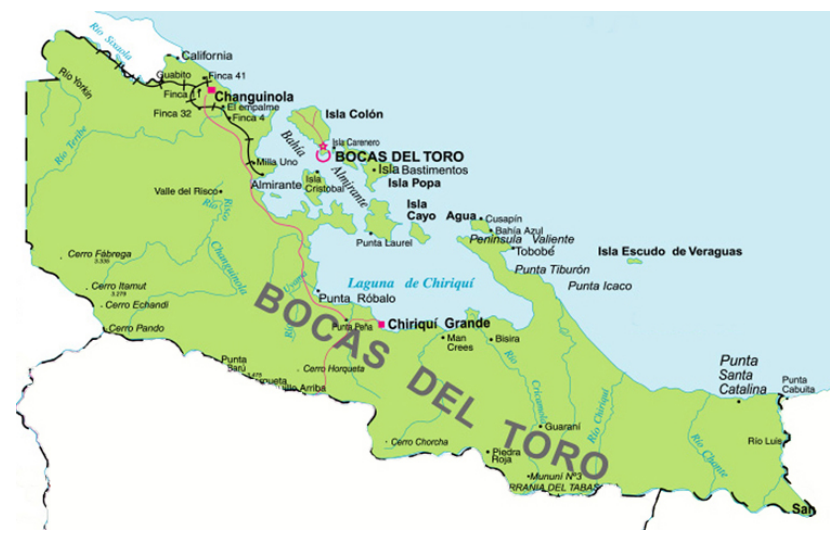

Figura 1. Mapa de la Provincia de Bocas del toro. Fuente: [6].

\subsection{Unidad de análisis y observación}

La unidad de análisis fue el distrito de Changuinola, y la unidad de observación fueron los moradores y consumidores de las áreas de El Empalme, El Silencio, Finca 6, Finca las 60, Finca las 30 y Guabito.

\subsection{Población y muestra}

La población del estudio estuvo conformada por 31,223 individuos, y la muestra de 380 usuarios de energía eléctrica del distrito de Changuinola, con un nivel de confianza de $95 \%$ $y$ un margen de error de $5 \%$.

\subsection{Variables de estudio}

La variable de estudio es la problemática en la red de distribución eléctrica. El Sistema de Distribución de Energía Eléctrica es la parte del sistema de suministro eléctrico cuya función es dotar de energía desde la subestación de distribución hasta los usuarios finales (medidor del cliente).

Los elementos que conforman la red o sistema de distribución son los siguientes:

$>$ Subestación de distribución: conjunto de elementos (transformadores, interruptores, seccionadores, etc.) cuya función es reducir los niveles de alta tensión de las líneas de transmisión (o subtransmisión) hasta niveles de media tensión para su ramificación en múltiples salidas.

$>$ Circuito primario.

$>$ Circuito secundario [11].

\subsection{Métodos e instrumentos de recolección de datos}

La recolección de datos se desarrolló utilizando la técnica de la encuesta, y como instrumento el cuestionario.

El cuestionario fue validado por un comité de expertos tomando como base las posibles causas de las fallas del fluido eléctrico. Por otro lado, se midió su confiabilidad utilizando
Alpha de Crombach, y un valor mínimo aceptable de 81. Además, se midió la confiabidad para algunos ítems con la prueba test retest, en donde los valores se encontraron arriba de 81, aceptando las preguntas con los ajustes solicitados. El cuestionario fue aplicado durante el mes de junio del 2018 a consumidores de energía eléctrica mayores de 18 años.

\subsection{Análisis de datos}

Los datos fueron procesados con ayuda del Programa SPSS versión 25 , con el cual, se realizó un análisis descriptivo de la variable principal, obteniendo tablas de frecuencia y gráficos de barras, que permitieron hacer explicaciones claras sobre la problemática presentada.

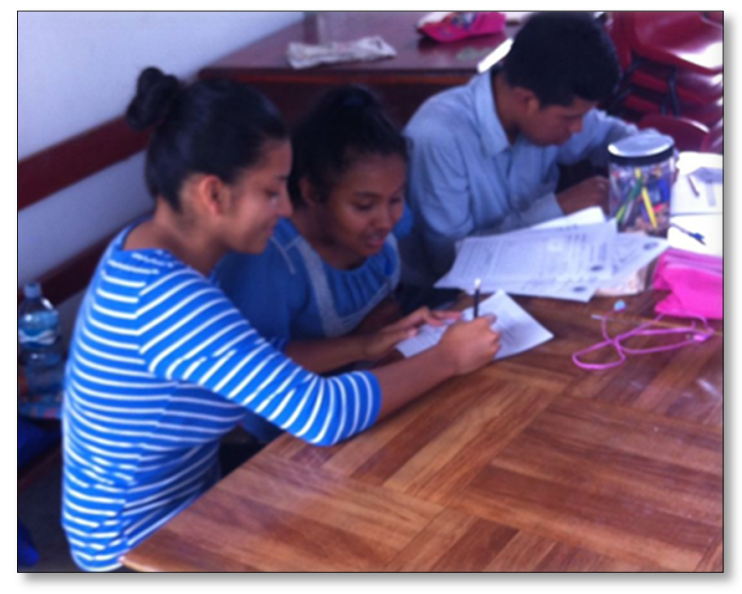

Figura 2. Aplicación de encuestas a moradores de Changuinola.

\section{Resultados y discusión}

Después de haber aplicado el cuestionario y analizado los datos, los resultados se muestran a continuación:

En la figura 3 se observa que el $30 \%$ de las personas afirman que se les interrumpe el fluido eléctrico una vez por mes, $42 \%$ señaló que sufre de apagones eléctricos cinco veces por mes y en un menor porcentaje de $28 \%$ explican que se va el fluido eléctrico más de cinco veces al mes.

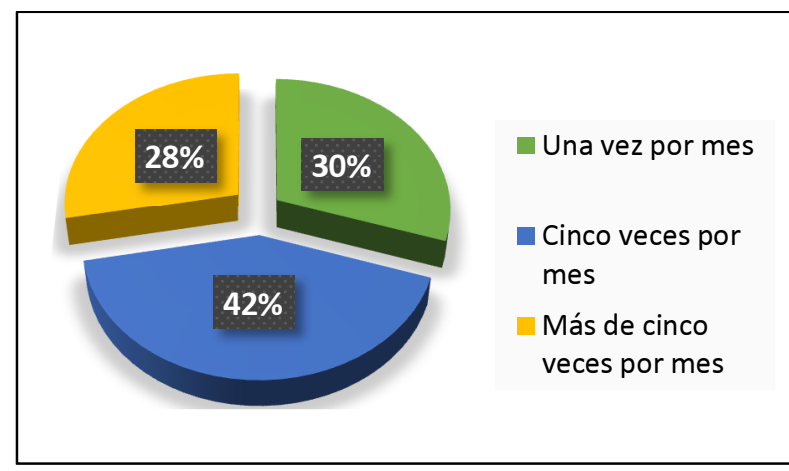

Figura 3. Frecuencia de las interrupciones del fluido eléctrico en el distrito de Changuinola 
Se sabe que cuando se presentan muchas interrupciones en el fluido eléctrico, los electrodomésticos tienden a sufrir daños, por lo cual, se quiso preguntar a la población si han sufrido pérdidas materiales debido a este fenómeno [12]. El resultado de las encuestas señaló que en el $74 \%$ de las personas no han tenido pérdidas materiales y un $26 \%$ dijo que sí han tenido pérdidas en sus electrodomésticos (figura 4). Esto, según explican, se traduce en pérdidas que sufren, porque los alimentos se descomponen y muchos aparatos eléctricos se dañan, dijo Venancio Caballero, presidente de la Cámara de Comercio de Changuinola [8]. Agregó el empresario que en el mencionado distrito hay 1,229 empresas, lo que constituye el $58 \%$ del total existente en la provincia, que de manera global tiene 2,032 [7]. Como es de notar la falta de un buen servicio no solo afecta a la población con viviendas sino a los comerciantes debido a que ellos tienen materiales que se pierden y en grandes cantidades.

Estudios de [13], indican que el almacenamiento de energía es indispensable porque la generación de electricidad a partir de energías renovables fluctúa.

Por otro lado, muchas familias, han tenido que salcochar sus alimentos, y consumirlos antes de lo planificado para evitar su pérdida [8].

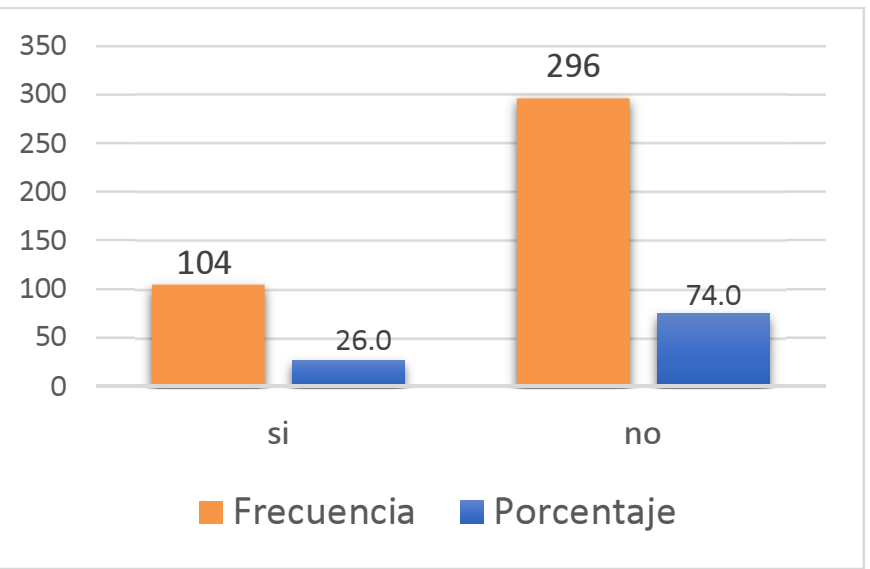

Figura 4. Pérdidas materiales debido a los constantes apagones.

Estudios de [14], explican que los estados deben fomentar y desarrollar las energías alternativas en sus territorios, por un medio ambiente sano y así, atender las necesidades prioritarias de energía de la población, pero sin alterar las condiciones ambientales.

La figura 5 muestra que el $26 \%$ de las personas creen que los daños son causados por la naturaleza, lo cual, indica que no tiene nada que ver con la empresa encargada de la distribución eléctrica. El 26\% de la población señala que los daños se deben a los cableados antiguos que existen en la actualidad por el área de Changuinola. Un 25\% cree que los daños son responsabilidad de aquellas conexiones que se realizan ilegalmente y sin pagar el costo debido de energía eléctrica.

El 16\% de la población opinó que las interrupciones son causadas por el hurto de los cables del tendido eléctrico, y el
$7 \%$ dijo que no sabían las razones de los apagones. Se destaca que el 3 de octubre de 2014, según [15] se registraron lluvias acompañadas de fuertes vientos que provocaron decenas de voladuras de techos en Miramar, Punta Robalo, El Empalme y la Isla Colón. Además, el tránsito en las carreteras Chiriquí Grande hacia Almirante y de Almirante hacia Changuinola, quedó imposibilitado debido a la caída de árboles, provocando fallas en el fluido eléctrico, apoyando las respuestas dadas en esta investigación.

Estos resultados promueven que estudios de [13], integrando redes inteligentes sean implementados, ya que, abarcan la integración de las tecnologías de la información para la infraestructura de la red eléctrica, en donde la gestión está dada en el control de equipos y cargas, en consecuencia, la operación automática del sistema, permite opciones productivas tanto en los operadores de servicios públicos como en los clientes.

Estudios de [16], explican que, para la compañía de distribución de electricidad, la integración de sistemas fotovoltaicos residenciales con una potencia instalada inferior a la calculada se implementaría fácilmente, logrando así minimizar la dependencia de los sistemas de generación eléctricas que presentan muchos problemas de fluctuaciones en las comunidades.

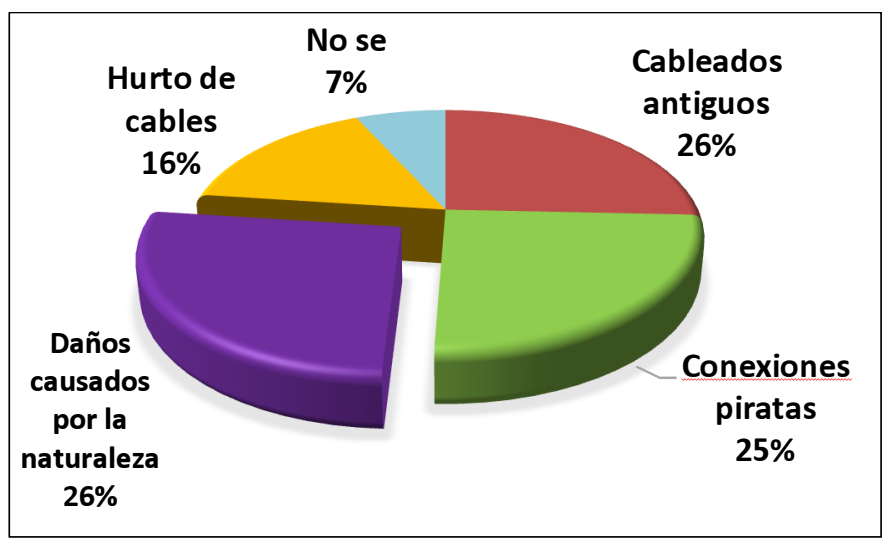

Figura 5. Probables causas de los continuos apagones eléctricos en Changuinola.

\section{Conclusiones}

Después de haber analizado los datos recolectados y la información obtenida, se puede concluir lo siguiente:

- El 42\% de los usuarios de energía eléctrica afirmaron que sufren de apagones eléctricos cinco veces por mes.

- Las personas casi no presentan pérdidas materiales debido a estos constantes apagones (26\%).

- El 26\% de los encuestados indican que los cableados de sus casas son antiguos, pudiendo ser la causa de los apagones eléctricos.

- El 26\% de los usuarios piensan que los apagones son causados por eventos naturales, como la caída de árboles, fuertes vientos y lluvias, pero son datos que no pueden asegurar. 


\section{AGRADECIMIENTO}

En primer lugar, le agradecemos a Dios por permitirnos culminar con éxito esta investigación. A nuestra asesora, la Dra. Vanessa Valdés por su gran ayuda y colaboración en cada momento de consulta y soporte en este trabajo.

A la Jornada de Iniciación Científica por la oportunidad y a la población del distrito de Changuinola, quienes colaboraron con las respuestas de los cuestionarios y a nuestras familias por su constante apoyo.

\section{REFERENCIAS}

[1] R. O. Argote Ramos, «100 años de historia de los servicios eléctricos en Panamá,» vol. II, nº 1, pp. 17-35, 2003.

[2] E. Inga , M. Campaña y R. Hincapié, «Optimal Sizing of Electrical Distribution Networks considering Scalable Demand and Voltage,» de 2018 IEEE 1st Colombian Conference on Applications in Computational Intelligence, Medellín, 2018.

[3] R. Baeza, J. Rodríguez y J. Hernández, «Evaluación de confiabilidad de sistemas de distribución eléctrica en desregulación,» Revista Facultad de Ingniería, U.T.A., vol. XI, nº 1, pp. 33-39, 2003.

[4] T. Horsley y J. Seymour, «Los siete tipos de problemas en el suministro eléctrico,» APC by Schneider Electric, vol. 18, $\mathrm{n}^{\circ}$ 1, pp. 2-5, 2016.

[5] R. Acevedo, «Desarrollo de la ingeniaería de sistemas de distribución como base para la modernización de empresas de servicio eléctrico,» II CONGRESO VENEZOLANO DE REDES Y ENERGÍA ELÉCTRICA, 2009.

[6] Distancia.km.net, «Distancia entre Changuinola y Ciudad de Panamá,» Programado por IGI., [En línea]. Available: http://distancia. $1 \mathrm{~km} . n e t / p a /$ changuinola $/$ pa/panama/. [Último acceso: 20 Mayo 2019].

[7] U. Vallejos, Interviewee, Gobernador de la provincia de Bocas del Toro. [Entrevista]. 18 Abril 2018.

[8] L. Machuca, «Se complica problema con sistema eléctrico en Bocas,» Panamá América S.A., 5 Febrero 2015.

[9] R. Hernández Sampieri, C. Fernández Collado y P. Baptista Lucio, Metodología de la Investigación, México: McGrawHill Education, 2015.

[10] Wikipedia, «Wikipedia,» WikiMedia project, 304 2018. [En línea]. Available: https://es.wikipedia.org/wiki/Provincia_de_Bocas_del_Toro . [Último acceso: 13 Mayo 2018].

[11] M. C. Castro, «Wikipedia,» WikiMedia Project, 17 Marzo 2018. [En línea]. Available: https://es.wikipedia.org/w/index.php?title=Red_de_distribu ci $\% \mathrm{C} 3 \% \mathrm{~B} 3 \mathrm{n}$ de energ\% $\% 3 \% \mathrm{ADa}$ el $\% \mathrm{C} 3 \% \mathrm{~A} 9$ ctrica\&acti on=history. [Último acceso: 13 Mayo 2018].

[12] L. M. G., «Se complica problema con sistema eléctrico en Bocas,» Panamá América, Cinco Febrero 2015.
[13] V. Gómez, C. Hernández y E. Rivas, «Visión General, Características y Funcionalidades de la Red Eléctrica Inteligente (Smart Grid),» Información tecnológica, vol. XXIX, nº 2, pp. 89-102, 2018.

[14] A. Bello y D. Paez, «Estudio de viabilidad financiera de la generación de energía eléctrica a partir de paneles solares Para puestos de salud en Colombia,» Facultad de Ingeniería, Bogotá, 2018.

[15] B. Lassonde, «Tempestad,» El Siglo, 3 Octubre 2014.

[16] A. Rios Villacorta, J. Luyo Kuong y D. Humpire Mojonero, «Impact Assessment of Net Metering for Residential Photovoltaic Distributed Generation in Peru,» International Journal of Renewable Energy Research, vol. VIII, n 3, pp. 1200-1207, 2018. 